\title{
Cloud-Based Synchronization for Interface Settings for Older Adults
}

\author{
Drew Williams, Sheikh Iqbal Ahamed ${ }^{1}$, William Cheng-Chung Chu* , Mong-Te Wang \\ 2 and Chih-Hung Chang ${ }^{3}$ \\ ${ }^{1}$ Marquette University, Milwaukee, WI, USA \\ ${ }^{2}$ Tunghai University, Taichung City, Taiwan \\ ${ }^{3}$ Hsiuping University of Science and Technology, Taichung City, Taiwan \\ ${ }^{1}$ drew.williams, sheikh.ahamed\}@mu.edu; ${ }^{2}\{c c h u$, shaka\}@thu.edu.tw, \\ ${ }^{3}$ chchang@hust.edu.tw
}

\begin{abstract}
When developing software, user interfaces are often designed with a genefal user in mind - which can result in applications with interfaces that are difficult to ase for those with different abilities. Older adult users, for example, often encounter impairment of their physical and cognitive abilities as they age, which means they look for different features in user interfaces when compared to a younger user. Adaptive interfaces can be incredibly helpful for users in these situations - rather han being forced to conform to an application's user interface offering, an older user canchange the user interface to match what works best for them. However, the increased user of mobile devices means that users often have more than one computing device, and thus, often are running more than one instance (or a mobile and desktop instance) of a particular applicalion. The use of cloud storage would be very beneficial in synchronizing settings from one instance of an application, across multiple devices. In doing so, we ensure that an application remains usable for an older individual across a wide variety of devices, and across platforms. In this paper, we explore the features such a cloud storage system would need to consider, in order to bring interface settings together across a vartety of connected devices and keep computers up to date with the users' preferred interface.
\end{abstract}

Keywords: human-computer interfaces, older adults, elderly users, cloud storage, software engineering

\section{Introduction}

The number of elderly individuals worldwide - that is, those aged 65 and older - is expected to double by the year 2025 [1], and thus the creation of proper interfaces for the elderly user has become a more and more important subject of discussion. As the number of older adults increases, additional effort is put forth to ensure their comfort and happiness. Computer applications have been noted to show great promise in assisting the elderly user [2], but existing applications can often be difficult to use for the older adult [3]. When creating applications, designers often target a general user - and in doing so, design an application that can be difficult to use for those with different abilities. Problems encountered by the elderly user can range from being forced to deal with difficult-to-understand graphical

\footnotetext{
* Corresponding Author: William Cheng-Chung Chu, cchu@thu.edu.tw
} 
interfaces $[4,5]$, to feeling lost when trying to use an application created without user tutorials [5]. As a result, several approaches making computers easier to use for elderly individuals have been developed - ranging from simplified applications [6] to complete computer ecosystems designed for the elderly user [7].

A particular solution suggested is that of adaptive interfaces, where settings allow an older adult computer user to customize the user interface for their particular needs. Such interfaces rely on user input for their data [8] - although in some cases, adaptive interfaces can automatically collect data [9] and even make intelligent decisions based on the data collected [10]. In any case, adaptive interfaces allow the user to customize an application's visual appearance to one that best fits their needs, making applications using this interface strategy understandable for a wider variety of users, while assisting the user in retaining their independence. However, this sort of solution has been complicated. More adult users than ever have not only multiple machines, but also mobile devices. In fact, the number of mobile devices is expected to exceed the number of people in the world within the year [11]. Many applications these days thus have multiple versions of particular applications, or at least access said application in two ways: via their desktop or laptop an̂d via their mobile device. Take into consideration the older adult user who uses applications using adaptive interfaces. If the elderly user chooses to carefully construct a series of settings for their particular needs within one instance of the application, what happens when the older adult moves to another instance of the application, either on his desktop or mobile device? The carefully configured settings would most likely need to be reconfigured all over again.

To fix this problem, a system of synchronization via cloud storage can be implemented to send and receive data across many devices. In addition to sending data about the interface to new machines where the older adult computer usec is using the application, if any changes or refinements are made to the interface settings at one of the other machines, these can be stored and synchronized with other machines using the interface data. This opens up opportunities for a user profile to be saved and - used across multiple applications, let alone multiple devices. However (ID order for such a system to work for the older adult user, several particular requirements must be met; the system must work in the background with little configuration required from the user, the system should be extensible and available, and the system must store data in a manner that leads the older user to trust it.

In designing such a system, we'll begin by giving our motivations for this project, in addition to going through some lelated work in human computer interfaces and cloud storage. After this we'll discuss our aforementioned requirements for the cloud storage system, and explain how to best create cloud storage system to synchronize settings information for elderly users with-more than one computing device. We'll finally evaluate our developed system and give a case study of it in use, offer our concluding remarks, and mention some future work to consider.

\section{Motivation}

In general, we seek to create applications that are more easily picked up by the older adult user - in this case, due to interfaces that adapt for the abilities of the older adult user, across multiple computers and platforms. Software in general can be very beneficial for older adult computer users; applications for email and social networking can help elderly users stay in touch with their friends and family, healthcare applications can assist older adults in monitoring their health, while various computer education systems can assist an elderly user in learning new things without registering for classes [2]. Adaptive interfaces, as mentioned, allow elderly adults to take advantage of these opportunities by creating a computer interface 
that they find simple to understand and use. Adaptive interfaces with added cloud synchronization for settings might then help further by extending user interface adjustments across computers, giving elderly users the ability to continue working with familiar interfaces even if they are unable to use their home computer, where they may have a series of specially-designed interfaces set up.

Our suggested interface also makes working with mobile devices a more attractive option for the older adult computer user. Mobile devices in particular have the potential to travel with the elderly user. In making the mobile device version of the application more familiar to the elderly user via a specially tailored interface (like the interface of the corresponding desktop application), we increase the chance of having the older adult user pick up the mobile application due to its increased familiarity - graphical user interfaces can often make or break applications. For example, take into consideration the following issues that may come up when an elderly user attempts to use a known application on a mobile device:

Improper or Inaccessible Visual Elements within an Application. Oftentimes, a mobile interface has been completely changed in order to efficiently use the screen space that exists for the app. This means that elements that may have been previously accessible, such as buttons or menus, could now be obscured and difficult to use. In addition, if an elderly user took a lot of time to perfectly configure an application to heir needs, and now finds they have to re-configure the mobile version of the application, their enthusiasm related to using mobile applications could wane (since they are faced with re-configuration for all the apps they might wish to use)!

Poor Ability to Interact with an Application. Many mobile applications rely on "intuitive" interactions with the interface, such as swipes or pinching motions. However, for an elderly user, these interactions may not be as intuitive as the developer thinks them to be which can cause usability problems within the application, and thus an aversion to using the mobile application.

Cognitive Overbad Related to an Application. Finally, while less common on a mobile device, the application could present so much information to the elderly user that they aren't sure what to concentrate on! While smaller screen space means that this is not necessarily an occurrence with the main interfice for a mobile application, push notifications and other popups and audio notifications certainly run the risk of causing such problems.

Finally, in the act of extending interface settings data across multiple devices, by using a cloud storage system mstead of something manual (such as moving settings via a USB drive, or via wireless synchronization such as Bluetooth connections), we can perform the data transfer required quickly as the data updated on the device. This ensures that including the data synchronization feature does not detract from an applications' usability.

\section{Related Work}

In order to understand the background for this sort of system, it's beneficial to explore related work across three fields: human computer interfaces for the elderly, adaptive humancomputer interfaces, and cloud storage. In this way, we can explore options that have been proposed for older adults who wish to use computers, the unique benefits of adaptive interfaces, and the current status of cloud storage (especially related to healthcare applications). 


\subsection{Human-Computer Interfaces for the Elderly}

As mentioned, due to the great benefits computers have for older adult users, a lot of work has been done in developing understandable and useable human-computer interfaces for the elderly user. The most common goal for these human-computer interfaces is to overcome age-related ability impairments that are known to occur in older adult humans. After all, a number of physical and cognitive problems are known to occur in old age [12], although it should be pointed out that said problems do not occur for all elderly adults [13].

The most common approach in attempting to fix these impairments is the development of 'elderly-friendly' computers, such as the Telikin, a ready-to-go computer with an interface designed to be simple to use for the older adult computer user [7]. Elderly-tailored software in particular is also quite common - Eldy is one such example, and transforms a typical desktop computer interface into a simplified screen consisting of several large buttons triggering a web browser, simplified chat software, and other useful applications [7]. Unlike the Telikin, Eldy is completely free and produced by a nonprofit organization [7]. There are even cases where very specific applications have simplified and recreated with "elderly-friendly" interfaces - in one particular project from the University of Dundee, the developers created an email client and web browser customized to the abilities of the elderly user [6]. The application included visual elements tailored for elderly users, a jargon-free interface, and minimal functionality [6]. In all cases, simplification is typically used to ensure that existing interfaces become digestible by the less computer-savvy elderly adult.

However, oftentimes this simplification becomes oversimplfication: as mentioned, the extent of the age-related changes a user may experience can vary. Developing a multitude of applications with a particular user abilitglevel in mind can affect how said applications are received - some users eschew applications "for the elderly" as a result of feeling as if the applications are over-simplifying thing for them [5, 13]. Among other reasons, older users may eschew these applications as they conflict With their need to feel socially included, by using the "normal" version of a software title [5] or simply due to wishing to reject the “elderly' label [13].

\subsection{Adaptable and Intelligent Human-Computer Interfaces}

Adaptable aser interfaces are particularly promising when developing for the elderly adult, as these interfaces have the capability to work for a variety of users. As would be expected from their name, adaptive interfaces can change themselves on the fly in relation to manual user input, or in some cases, collected data about users' patterns of using the application. These interfaces may be used for desktop applications, used in web applications, or even in mobile applications.

Dickinson et al., (2007) created a customized search and navigation system for the elderly adult computer user, which allowed for a hierarchy of three levels of content access: a very simplified interface for users who were unfamiliar with computers or otherwise desired a straightorward interface, an interface that included limited material that had not been simplified, and a third layer created without accessibility concerns [14]. Magee and Betke (2010) developed a hierarchical web browser that also provides the user with a number of levels; each corresponding to increasing complexity of the UI, thus allowing the user to pick the level they felt was appropriate for their needs [8]. SUPPLE builds a model of the users' preferences through the use of preference statements composed by the user, rearranging its interface to match what best fits the preferences of the user [9]. The sequel project, SUPPLE++, allows for the modeling to occur on the fly after the user works through a series of performance assessment tasks [9]. A similar system proposed by Leiva (2012) changes the 
interface of a website based on users' actions taken in navigation, to help the website best suit their individual navigation styles [15]. Adaptive interfaces in mobile applications are also being used to teach and adapt to the needs of elderly mobile users [16].

\section{Table 1. Comparison of Different Interfaces for Elderly Computer Users}

\begin{tabular}{|c|c|c|c|}
\hline Type of Interface & Interface Example & Positive Qualities & Negative Qualities \\
\hline $\begin{array}{l}\text { Customized Human- } \\
\text { Computer Interface: } \\
\text { A simplified interface } \\
\text { that remains the same } \\
\text { for all users. }\end{array}$ & $\begin{array}{l}\text { Eldy, a computer } \\
\text { interface designed for } \\
\text { the abilities of the } \\
\text { elderly computer user. } \\
\text { [7] }\end{array}$ & $\begin{array}{l}\text { Simplifies existing } \\
\text { interface [7], prevents } \\
\text { cognitive overload that } \\
\text { may occur with typical } \\
\text { interfaces. }\end{array}$ & $\begin{array}{l}\text { Does not greatly adapt } \\
\text { for individual user } \\
\text { preference. }\end{array}$ \\
\hline $\begin{array}{l}\text { Adaptive Human- } \\
\text { Computer Interface } \\
\text { An interface that } \\
\text { changes based on user } \\
\text { preference }\end{array}$ & $\begin{array}{l}\text { HAIL, a web browser } \\
\text { with a number of levels } \\
\text { of interface complexity } \\
\text { for users with } \\
\text { disabilities. [8] }\end{array}$ & $\begin{array}{l}\text { Simplifies interface, } \\
\text { Adapts for the } \\
\text { individual }\end{array}$ & $\begin{array}{l}\text { Adaptation is not } \\
\text { automatic, } \\
\text { aser inpuires as to which } \\
\text { level of adaptation they }\end{array}$ \\
\hline $\begin{array}{l}\text { Intelligent Human- } \\
\text { Computer Interface: } \\
\text { An interface that } \\
\text { changes based on } \\
\text { decision-making } \\
\text { algorithms in } \\
\text { application. }\end{array}$ & $\begin{array}{l}\text { SUPPLE++, which } \\
\text { automatically models } \\
\text { user ability, based on a } \\
\text { series of performance } \\
\text { assessment tasks. } 97\end{array}$ & $\begin{array}{l}\text { Adaptation is based not } \\
\text { on pre-existing/pre- } \\
\text { programmed levels of } \\
\text { user ability, but reacts } \\
\text { to user skill shown } \\
\text { during performance } \\
\text { assessments. [9] }\end{array}$ & $\begin{array}{l}\text { Requires moderate use } \\
\text { of the application } \\
\text { before creating a } \\
\text { settings profile for the } \\
\text { user, may not be } \\
\text { preferred by the user } \\
\text { due to this exertion. [9] }\end{array}$ \\
\hline
\end{tabular}

\subsection{Cloud Computing}

Cloud computing was recently defined by the National Institute of Standards and Technology as "a model for enabling ubiquitous, convenient, on-demand network access to a shared pool of configurable computing resources." [17] All cloud computing instances, regardless of their service and deployment models, share five particular features: on-demand self-service, broad networkaccess, resource pooling, rapid elasticity and measured service. [17] While a variety of consumer applications using cloud storage technology exists, such as personal online storage applications [18] and document-specific storage and creation programs [19], healthcare applications using cloud storage are being looked into as well. Among other things, cloud storage can be used to deliver on-demand healthcare services to older adu ts [20], improve the quality of healthcare being offered with limited healthcare resour es [21], and access the prescriptions of a particular user to monitor for adverse reactions to medication combinations [22].

\section{Requirements for Cloud Storage Systems for Interface Settings Transfers}

In order for our idea to succeed, we need to fulfill particular requirements to ensure that the system is easy to use, understandable and extensible for both the patient and the practitioners involved. Without these traits, the elderly user may not take advantage of the cloud synchronization abilities, due to not being able to understand how to use the system or not 
trusting the security of the system. Additionally, a practitioner might find the data secured to be not easily transferred or not applicable to other applications. This said, here are some of the requirements for our cloud storage system that we should take into consideration.

\subsection{Easily Configurable Data Transfer}

The first requirement is that the data transfer process not be problematic for a person with limited experience with computers to initiate. If a user is asked to re-initiate the data transfer to the cloud, or otherwise think about the process of transferring data, they run the risk of forgetting to upload data to the servers - meaning that they will have to re-populate settings themselves the next time they load the application. If this happens multiple times, it ultimately defeats the purpose of our system, which is to ensure that an elderly user can easily access their settings across devices without a problem.

\subsection{Available and Ready Access to Data}

The next requirement is that the system should be relatively available for older adult computer users to upload new data to. This rules out something such as a networked drive synchronization system, where an elderly user would-need to be at home in order to add or synchronize new data - using a cloud storage system ensures that new settings data can be uploaded no matter where the user is. However, it should be known that the unpredictability of external circumstances means that one can ne er assume a $100 \%$ uptime for cloud systems - unplanned maintenance is always a factor.

\subsection{Extensible Access to Data}

In order to get the most out of the proposed system, the data shouldn't be stored in a proprietary database or in a format that only one software company can access. Having the setting data in an open format allows for other applications to use the data to set their own user interfaces - potential Yoreating a network of applications that all utilize and add to settings data for an॰ older adult computer user, significantly enhancing their computing experience across a variety of applicatrons!

\subsection{Secure Data Transfer?}

Finally, in order for the program to be trusted by the older adult individual, the system should be relatively secure. Although applications settings data may not be at first glance considered data tha could harm an individual if accessed by an unauthorized person, consider that if it indicated over time that a older adult computer user's eyesight was getting worse, this data does have the potential to harm the users' personal life. Furthermore, an explicit understanding of what is being gathered by the software is imperative; older adults are often quite concenned about their privacy when using applications [5], so being up-front about the sort of data being collected can help them feel more at ease with allowing the settings data to be collected.

\section{Developing a Cloud Storage System for Interface Settings for Older Adults}

Our requirements stated, we can now set about developing the proper cloud storage system for saving and synchronizing our user interface settings. Data first enters our system and cloud storage via an initial set up - which is when our older adult user first installs an 
application using our synchronization system. Since we expect multiple applications to be using this synchronization system, the very first application will ask an older adult user to create a login username and password, and create a user profile for the individual. The user profile will be stored in an encrypted state on the computer, on the cloud server, and transmitted using a secure data transfer protocol - however, because physicians may use the data or be required to view the data to troubleshoot it, it should not be encrypted in a clientside matter, i.e., where administrators for the synchronization service did not know the private key to unlock the data. This could be an option in future iterations of the synchronization service if user interest in the capability was high, although as mentioned some functionality in terms of data aggregation and reporting may be lost.

Settings will begin in a default state. However, the applications using this cloud storage system - no matter their platform - should make it explicitly understood how to change the settings. This way, an older adult computer user - who may not have much experience with using computers - can take advantage of this system without it causing them too much stress. A jargon-free settings menu is the best way to initiate this. The older adul computer user, who may not know what terms such as "icons" and "resolution" are referring to, often finds technical vocabulary and jargon confusing. By creating settings menus that initiate via a user asking for help, and then ask a user if they can see the screen clearly of hear their music clearly - the older adult computer user can more easily change theiy settings preferences without outside help. Another way to initiate this requirement is to initiate a weekly questionnaire that asks the user about various patts of the application, and its respective usability - for example, if the buttons shown on creen are easy to click.

Now that we have settings data being fed into the application, we need to aggregate the data being received into a user profile. These user profiles are what we're ultimately going to be synchronizing, as the steps to make an application accessible for a older adult computer user with demonstrated poor vision of difficulty pressing a particular button will more than likely be different on a desktop computer and on a tablet computer. User profile data will indicate not only some techmical preferences, such as a user preference for a font size of 20, but make assessments about the older adult based on the settings fed into the system. For example, if an oldet adult user refimes an application to have a bigger font size and higher contrast by indicating that they can't read the app's text and they cannot see the text because of the color chorce, the system records both their choices for text color and font size, and that the older adult has a particula seore in relation to how good their vision is.

A users' vision, hearing, cognitive abilities (i.e., how overwhelmed they feel by receiving notifications) and moreceach receive these individual scores, which feed into a profile that gives an overall picture of what sort of interface the user prefers. By passing along these scores into cloud storage, in addition to particular data about interface preferences, applications on different platforms can adjust in anticipation of a users' needs by looking at the scores and the settings data particulars, and deciding which is easier to conform to. For example, if a user records a preferred text size of 20 , and then decides to use their cloudstored settings data with a mobile phone application, the mobile phone application may deem it unhelpful to up the text size to 20 on such a small device - but they can increase text size to a particular extent in response to seeing that the user has poor vision!

Now, the path of the data within the system after it has been populated can be seen in Figure 1. After the initial profile is created and settings data is uploaded to the cloud, changes in this settings data will initiate changes to the cloud-stored data - keeping the cloud-stored data up to date with current user preferences. If a user logs into an application on a secondary device or a mobile device that also uses this cloud storage synchronization platform, they will initially download the settings data in the cloud instead of reverting to defaults for settings on 
their device. Data will continue to upload and update the cloud-stored data across all devices; if a user makes a change to volume or determines that their buttons are too small on a mobile device, the changes trickle down to the cloud-stored settings and resultant changes to volume or button size may be made in their desktop applications. A change in the cloud-stored settings data prompts updates to all applications currently using the cloud-stored settings.

\section{Evaluation of the System}

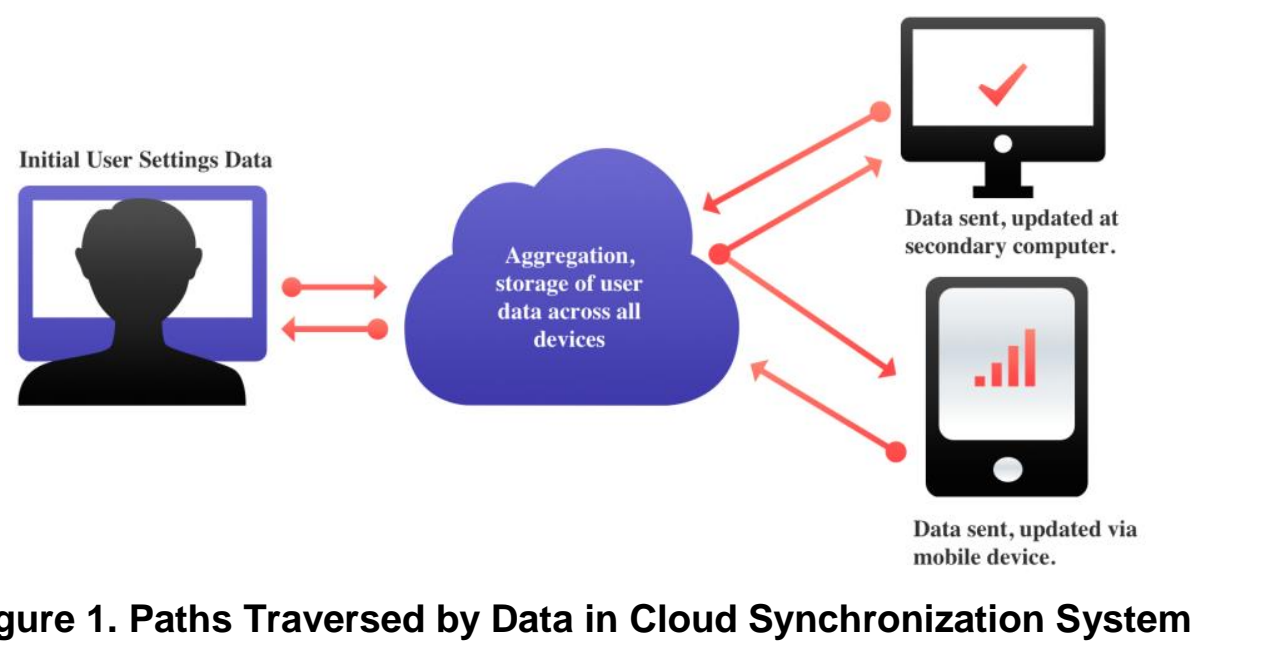

The most important thing to consider about this system is that it allows an elderly user to spread one set of settings across muliple applications and devices - older adult users do not need to implement individualized settings muitiple times across multiple applications and devices any longer. If an elderly user was tavelling, they could use a local library's computer and feel as at home with the interface as they do with their own home machine. This in turn relieves some of the frustration an older user may experience when using a computer, and thus makes the use of computers more appealing to older adult computer users. It also would make the use of moble devices more appealing: difficulties amended on desktop machines would be in turn amended on mobile machines. A side effect of the storage system, the jargon-free settings menus required for easy settings management on part of the user, would also ensure that users Nould be able to manipulate their settings skillfully without having to parse unfamiliar vocabulary.

Our system also fully complies with our requirements previously listed in section four; data stored in the cloud synchronization system does not require any additional work on the part of the computer user to be transferred, and is very available, extensible, and secure.

\subsection{Automatic, Background Data Transfer}

Cloud storage data transfer is typically configured to occur in the background, without user intervention, when data in a system is updated. In this system's case, this means an older user does not need to initiate the data transfer, and transfers will continue as data is updated without user intervention - all that may be required is a simple login process before a user can access their settings data. This makes the system easy for older adult computer users who may have little experience with computers; they don't need to worry about any additional work in storing their interface settings in the cloud. 


\subsection{Available Access to Settings Data}

Storing settings in the cloud ensures that access to a user's settings will be available, no matter what device a user is using and where they are. If an elderly individual decides to download a new coupon application on their mobile device in a supermarket after seeing an advertisement for it, and it uses this cloud synchronization framework, the settings data can be accessed and applied immediately. Furthermore, settings data is backed up in case of computer or mobile device failure. Dropping one's phone would not destroy any carefully constructed settings for their mobile environment; after logging into the synchronization platform on their new device, all of the data would be repopulated.

\subsection{Extensible Access to Data}

This cloud storage synchronization system is also an optimal solution because it ensures the extensibility of the system - both across applications and across devices. Storing the data in a database that can be accessed via web service calls or something simllar ensures that multiple applications can access the data and use it for devices running different operating systems. The importance of extensibility goes beyond being able to use existing settings data with different applications, however. In particular, settings data - as mundane as it may seem - could be used to map increasing difficulties in older adult users over time, and possibly predict the onset of cognitive disorders or physical disease. If a user's eyesight is steadily growing worse over a month-long period, it nay be indicative of an underlying illness. Storing this data in an accessible databas allows for uses like this to be explored as necessary.

\subsection{Data Security: in Transit and Storage}

Finally, as mentioned, steps will be takên logensure that the data gathered is secure in storage and in transit. When stored on-device and in the cloud, data will be secured using encryption - only secure methods of transmission will be utilized regarding the data as well. While older individuak may be a bit concerned due to the fact that the data would be accessible by administrators while being stored in the cloud servers, explicitly stating that exact user activities are not recorded and transmitted, and the data that is gathered is only used to ensure a user has properly configured computing environment, should help in assuaging user fears. While indeed the uses of the data may change in the future, as mentioned regarding mapping settings data to predict the onset of illness, measures must be taken in these casesto inform the user of any additional data uses and above all, ask their permission in using their interface data to make predictions about their health.

\subsection{Case Study: Using Cloud-Stored Synchronization in Traffic Applications}

To walk through an example of this synchronization system in use, we can take as an example the application "MetroMove", which allows a user to map routes to locations in their city and assess the best method of arriving at said destination based on current reported traffic and nearby bus schedules. As such an application would certainly be useful to elderly individuals, the application uses this cloud storage synchronization system to assist in being user-friendly for older adult computer users. In this way, it can ensure that its interface matches the needs of the user who happens to download the application, rather than forcing the user to adapt to a "general" interface implemented by the developers.

Michelle, a 65-year-old teacher, decides to download MetroMove to see if she would be better off taking a bus to work rather than driving on days when there is heavy traffic. She 
downloads the application to her phone first, and opens it as soon as it is done installing. Since Michelle has never used an application using this cloud-stored settings system before, she makes an account when the opens the application for the first time. This initializes the storage of her settings information: a folder is created on her phone to store the data, which is then uploaded to the cloud server periodically in the background. Michelle never receives obtrusive notifications regarding this data upload, unless something has gone terribly wrong (i.e., no data connection for several days, failed/corrupted upload).

After she starts the application, she notices that some of the text on the buttons is far too small to see. Because MetroMove uses the default settings on first start, she needs to edit these settings so they will be tailored to her liking. She selects a question mark icon with "Help" written beneath it in the application's toolbar, and is asked if she needs help with using the application or with changing how the application looks. She walks through several plainly worded, non-technical questions before she can state that she can't use certain butons in the application because the text is too small. MetroMove resizes the buttons to make them slightly bigger, logs her button size preference, and logs that her eyesight is below average. This is uploaded to the cloud, along with similar changes as over the mext day or two, Michelle customizes the application to her needs.

Now, Michelle finds that the application is fantasticat saving her time in navigating traffic in the morning, and deciding if she should just take the bus Later, she discovers that MetroMove has an accompanying website, where one can log into the settings synchronization system and the appearance of the websiteschanges to what a user is familiar with. She boots up her desktop, accesses the website and is greeted with a box asking if the website can access her settings data - after all, it does require authentication to use. She agrees, logs into her account, and the MetroMove vebsite accesses her user settings data, and manipulates its appearance in line with her preferred font sizes and button sizes, in addition to the notes about her eyesight being below average. When the website loads, it has a highercontrast view, large buttons (notas large as her phone, due to the assumed keyboard and mouse setup, but more visibie) and larger font size. She can now make her maps on a larger screen, and print them! Her grandkids are thrilled that she is finally using her computer regularly, and Michelle can now plot routes all around her neighborhood via the bus systems without wading through the less-understandable map applications' interfaces.

\section{Conclusion}

In conclusion, this synchronization system shows potential in assisting in the creation of an ideal elderly-friendly user interface. Creating a centralized user profile allows information to be aggregated about the older adult user's preferences regarding their user interface, and these preferences to be integrated across different applications running on different operating systems. The data is extensible due to being stored in an open format, and available, and stored and transmitted securely. Future work in this area may include adapting the settings data in the presence of different input or interface interaction technologies - if a user had a touchs reen computer, and noted a problem with their vision, buttons may need to change color and size in a different manner when compared with a typical computer using a mouse and keyboard input system. Detailed customization such as this is key to creating properly adaptable interfaces, for the elderly population and others.

\section{Acknowledgements}

This work is sponsored by TUNGHAI UNIVERSITY 'The U-Care ICT Integration Platform for the Elderly - No.102 GREEnS004-2', 2013. 


\section{References}

[1] G. Sealey, U.S. Elderly to Double in 25 Years, ABC News, Available: http://abcnews.go.com/US/story?id=91943\&page=1, (2014).

[2] N. Alm, P. Gregor, and A. F. Newell, "Older People and Information Technology are Ideal Partners", International Conference for Universal Design, (2002) November/December; Yokohoma, Japan

[3] D. Williams, M. A. Ul Alam, S. I. Ahamed, and W. Chu, "Considerations in Designing Human-Computer Interfaces for Elderly People", Proceedings of the 13th International Conference on Quality Software (QSIC), Edited A. Gotlieb and Z. Chen, Nanjing, China, (2013) July 29-30.

[4] L. Kantner and S. Rosenbaum, "Usable Computers for the Elderly: Applying Coaching Experiences", Proceedings of the 2003 IEEE International Professional Communication Conference, Orlando, Florida, USA, (2003) September 21-24.

[5] S. Sayago, D. Sloan, and J. Blat, "Everyday Use of Computer-mediated Communication Tools and Its Evolution over Time: An Ethnographical Study with Older People", Interact Comput, vol. 23, no. 5, (2011), pp. 543-554.

[6] A. F. Newell, "HCI and Older People", HCI and the Older Population Workshop at British HCI, (2004) September 7; Leeds, UK.

[7] J. T. Miller, "Simplified Computer Software That Can Help Seniors Get Online", Huffing on Post, Available: http://www.huffingtonpost.com/jim-t-miller/software-for-seniors_b_1852656.html, (2012).

[8] J. Magee and M. Betke, "HAIL: Hierarchical Adaptive Interface Layout, Lecture Notes in Computer Science", Edited by K. Miesenberger, J. Klaus, W. Zagler, and A. Karshmer, Springer Berlin Heidelberg, vol. 6179, (2010), pp. 139-146.

[9] K. Z. Gajos, J. O. Wobbrock, and D. S. Weld, "Improving the Performance of Motor-Impaired Users with Automatically-Generated, Ability-based Interfaces", Proceedings of the SIGCHI Conference on Human Factors in Computing Systems, Florence, Italy, (2008) April 5-10, pp. 1257-1266.

[10] A. Hunter, H. Sayers and L. McDaid, "An Evolvable Computer Interface for Elderly Users", HCI Conference on Workshop Supporting Human Memory with Interactive Systems, Lancaster, UK, vol. 204, (2007) September 4.

[11] Mobiles 'to outnumber people next year', sâys Un agency, BBC News, Available: http://www.bbc.co.uk/news/technology-22464368, (2013).

[12] T. Phiriyapokanon, "Is a Big Button Thterface Enough for Elderly Users?", Masters' Thesis, Malardalen University, Sweden, (2011).

[13] J. Durick, T. Robertson, M. Brereton, F. Vetere and B. Nansen, "Dispelling Ageing Myths in Technology Design", Proceedings of the 25th Australian Computer-Human Interaction Conference: Augmentation, Application, Innovation, Collaboration, Sydney Australia, (2013) December 2-5.

[14] A. Dickinson, M. J. Snith, J. L. Arnott, A. F. Newell and R. L. Hill, "Approaches to Web Search and Navigation for Older Computer Novices", Proceedings of the SIGCHI Conference on Human Factors in Computing Systems, San Jose, California, (2007) April 28-May 3.

[15] L. Leiva, "Interaction-based User Interface Redesign", Proceedings of the 2012 ACM International Conference on Intelligent Use Intêrfaces, Haifa, Israel, (2012) February 24-27.

[16] C. Bruder, L. Blessing an H. Wandke, "Adaptive Training Interfaces for Less-experienced, Elderly Users of Electronic Devices”, Benav hif Technol, vol. 33, no. 1, (2014), pp. 4-15.

[17] N. US Department of Commerce, "Final Version of NIST Cloud Computing Definition Published." Available: http://www.nist.gov/itl/csd/cloud-102511.cfm.

[18] "Dropbox,” Dropbox. [Online]. Available: https://www.dropbox.com/.

[19] "Overview of Google Drive - Drive Help." [Online]. Available: https://support.google.com/drive/answer/2424384?hl=en\#2424384.

[20] Y.-Y. Ou, P.-Y. Shih, Y.-H. Chin, T.-W. Kuan, J.-F. Wang and S.-H. Shih, "Framework of Ubiquitous Healthcare System Based on Cloud Computing for Elderly Living", 2013 Asia-Pacific Signal and Information Processing Association Annual Summit and Conference (APSIPA), Kaohsiung, Taiwan, (2013) October 29November 1 .

[21] Fujitsu Limited, Fujitsu Introduces Cloud Service for Senior Care, Fujitsu Global. Available: http://www.fujitsu.com/global/news/pr/archives/month/2013/20130123-01.html, (2013).

[22] B. T. Horowitz, "Researchers Enlist IBM Cloud In Quest For Better", Safer Elder Care, CruxialCIO. Available: http://www.cruxialcio.com/researchers-enlist-ibm-cloud-quest-better-safer-elder-care-7476, (2014). 


\begin{abstract}
Authors

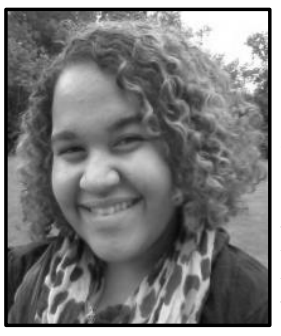

Drew Williams, a graduate student at Marquette University, where she is working toward her $\mathrm{PhD}$ in Computational Sciences and working as a research assistant and lab manager of Marquette's Ubicomp Lab in the department of Math, Stat. and Computer Science. In May of 2014 she finished her Master of Science in Computing, concentrating on human-computer interfaces for the elderly computer user for her thesis; prior to this she completed her Bachelor of Science in Computer Science at the University of Chicago. In addition to research interests in human-computer interfaces, Drew's research interests include ubiquitous computing, augmented reality and the development of mHealth applications.

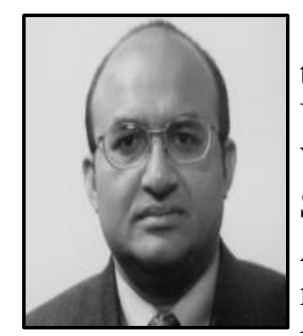

Sheikh Iqbal Ahamed, a professorand director of Ubicomp Lab in the department of Math, Stat., and Computer Science at Marquette University, USA. He is also a Faculty member of Medical college of Wisconsin, USA. He is a senior member of the IEEE Computer Society and ACM. He completed his Ph.D. in Computer Science from Arizona State University, USA in 2003 Fis research interests include mHealth, security and priy acy in pervasive computing and middleware for ubiquitous/peprasive computing. He has published 100+ peer reviewed journal, conference and workshop papers including seven best paper/posters. Dr. Ahamed serves regularly on international conference program conmittees in software engineering and pervasive computing such as COMPSAC '13, COMPSAC '12, PERCOM '08, and $S A C$ ' 08 . He is the Guest Editor of Computer Communications Journal, Elsevier.

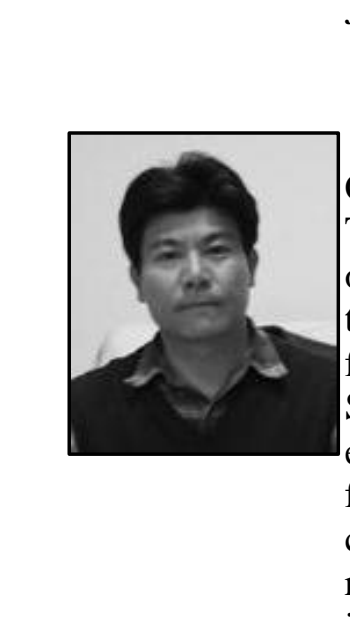

William Cheng-Chung Chu, a professor of the Department of Computer Science, and the Director of Software Engineering and Technologies Center of Tunghai University. He had served as the Dean O1) Research and Development office at Tunghai University from 2004 to 2007, and the dean of Engineering College of Tunghai University from 2008 to 2011, Taiwan. He is member of the IEEE Computer Society. His current research interests include software engineering, embedded systems, and E-learning. Dr. Chu received his $\mathrm{PhD}$ degrees from Northwestern University in Evanston Illinois, in 1989, in computer science. He has edited several books and published over 100 referred papers and book chapters, as well as participating in many international activities, including organizing international conferences, serving as steering committee for COMPSAC, APSEC and the program committee of more than 70 international conferences. His email address is cchu@thu.edu.tw.
\end{abstract}




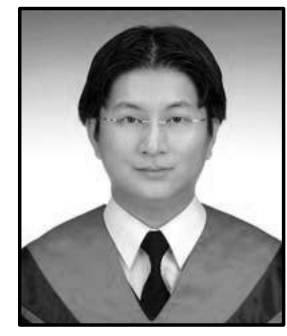

Meng-Te Wang, a Research Assistant in Software Engineering \& Technology Center of Tunghai University(2004-2014). He majored in Computer Science, and graduated with a Master's degree at Tunghai University in May, 1998. Besides human-computer interfaces, system Simulation, bioinformatics and Stat., Mong-Te Wang's research interests include object-oriented technology, software re-engineering, requirement modeling of embedded system and the development of mHealth applications. His email address is shaka@thu.edu.tw.

Chih-Hung Chang, Ph.D., received his Ph.D. degrees in Computer Science from Feng Chia University in 2004. Currently, he is an associate professor at Hsiuping University of Science and Technology. $\mathrm{He}$ is member of the IEEE Computer Society. His research interests include object-oriented technology, software re-engineering, and requirement modeling of embedded system. He has published more than 100 papers in international journals and conferences. He served as local arrangement co-chair for SAC 2011 and COMPSAC 2015, and co-chair for SQHE'13, SAC 2011 APGCC Track, and WESQA'10. His email address is chchang@ @ust.edu.tw.

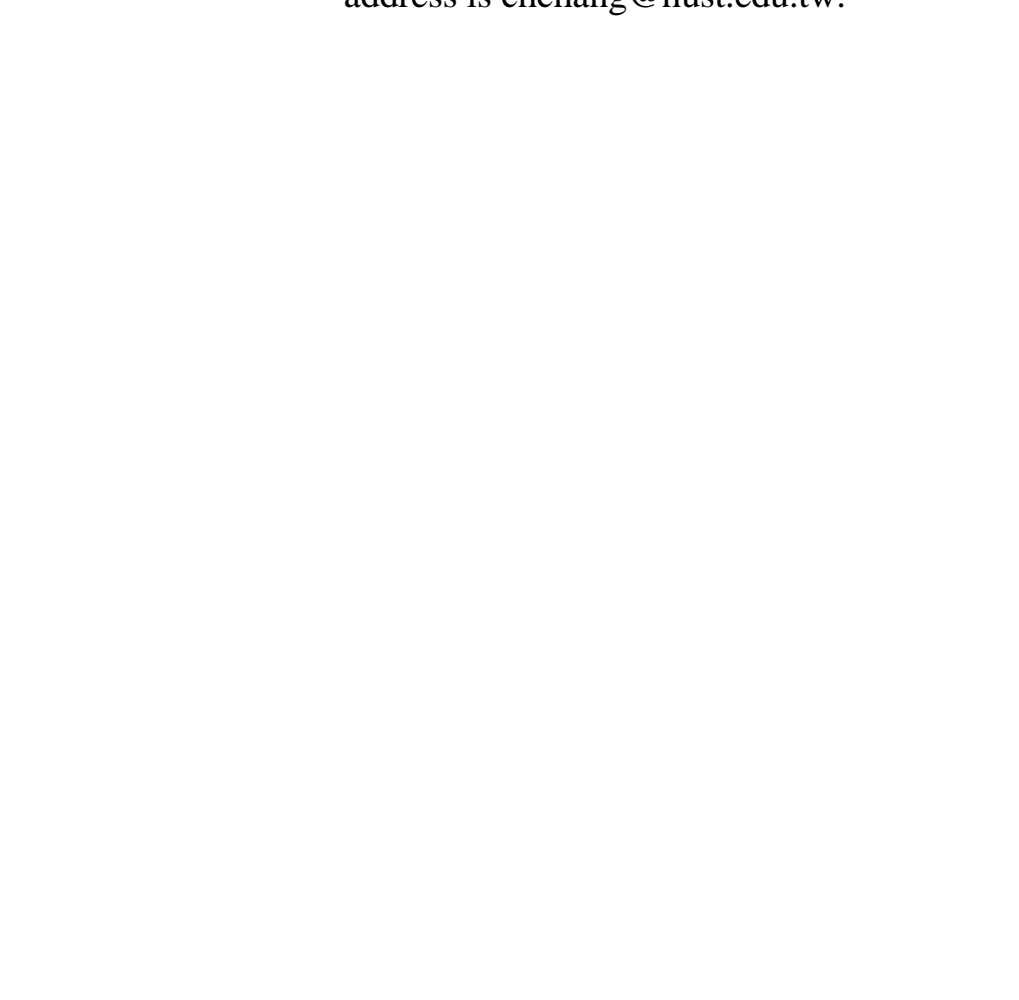


International Journal of Hybrid Information Technology

Vol.7, No.4 (2014)

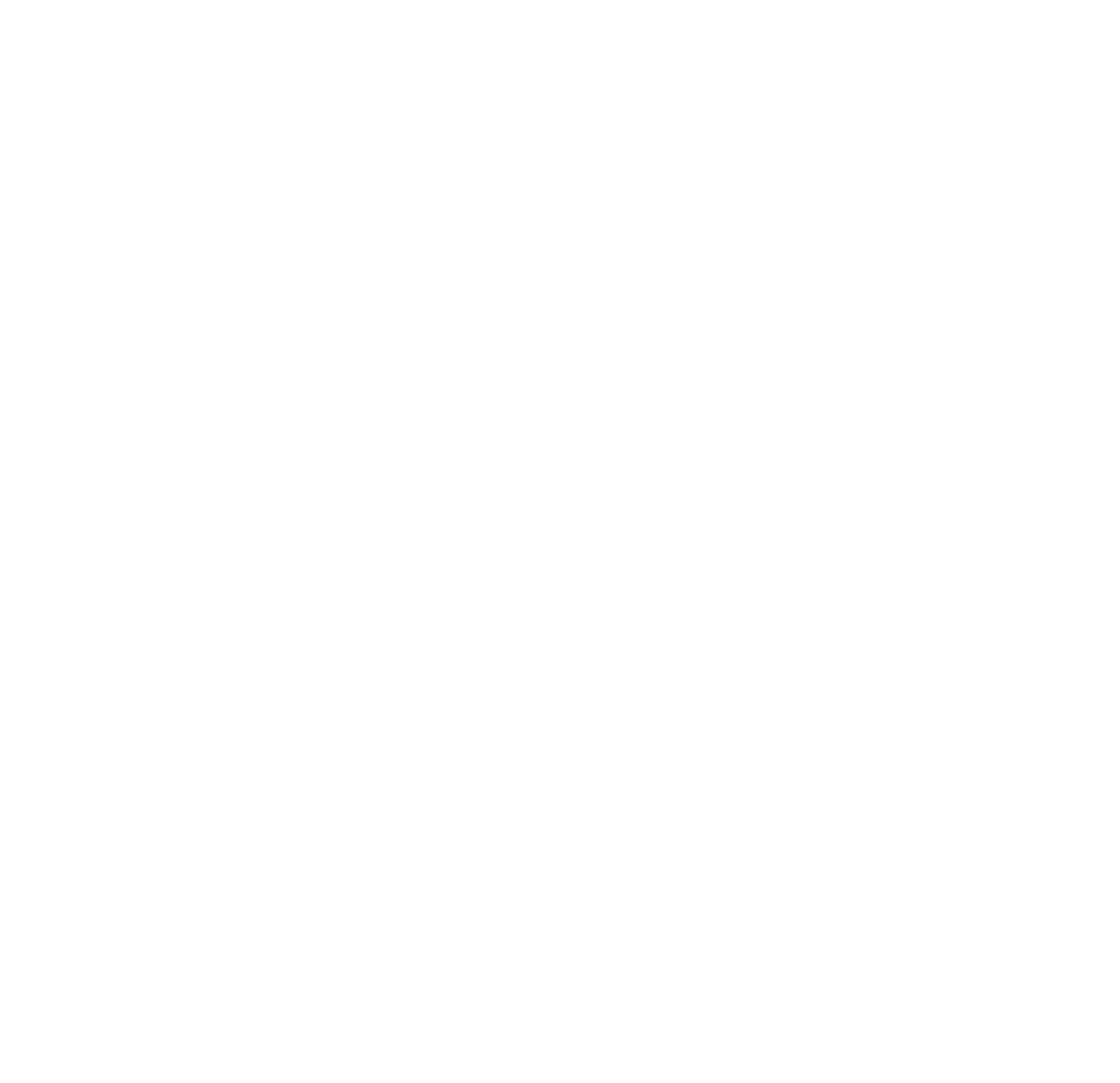

